\title{
Directive de l'ASSM sur l'attitude face à la fin de vie et à la mort
}

\section{Philippe Luchsinger}

président de mfe Médecins de famille et de l'enfance Suisse

\begin{abstract}
La directive sur l'attitude face à la fin de vie et à la mort a été approuvée et publiée par l'ASSM. Elle devra être intégrée dans le Code de déontologie de la FMH lors de la prochaine chambre médicale. Le Comité central de la FMH et le Comité de direction de la CCM s'y opposent, mfe médecins de famille et de l'enfance Suisse a interrogé ses délégués et soutient la directive de l'ASSM.
\end{abstract}

Le débat sur la directive élaborée et publiée par l'ASSM sur l'attitude face à la fin de vie et à la mort a été particulièrement houleux. Une directive qui, comme toutes les autres, a dû faire l'objet de modifications pour s'adapter à la société actuelle. Dans le cadre d'une procédure mise en place avec soin, l'ASSM a procédé à une actualisation en conséquence impliquant des personnalités du secteur de la santé très versées sur l'éthique, et, en particulier, des médecins du domaine des soins palliatifs, qui ont collaboré au processus en toute conscience et en toute responsabilité. Par ailleurs, tous les cercles intéressés ont été appelés à s'exprimer dans le cadre d'une consultation. La grande majorité des organisations ont approuvé la directive avec de légers ajustements. mfe et la CMPR ont également fait connaitre leur accord. Le résultat est une directive traitant des sujets sensibles avec beaucoup d'égard et de prudence.

Le principe et pratiquement tous les points de la nouvelle directive sont incontestés. Un point a soulevé un vif débat: faut-il autoriser les médecins à accompagner

À plusieurs reprises, le peuple suisse a indiqué très clairement qu'il apprécie, mais également attend, un soutien en cas de désir de mort prématurée.

un(e) patient(e) dans une procédure de suicide volontaire même s'il ne souffre pas d'une maladie mortelle mais que son état lui fait considérer que sa vie ne vaut plus la peine d'être vécue.

À plusieurs reprises, le peuple suisse a indiqué très clairement qu'il apprécie, mais également attend, un soutien en cas de désir de mort prématurée. Il est également évident pour la population que cette démarche doit faire l'objet d'une réflexion approfondie et responsable, et que sa mise en œuvre doit s'effectuer avec délicatesse et d'un commun accord. C'est précisément l'objet de la directive sur l'attitude face à la fin de vie et à la mort: il faut que le souhait d'un(e) patient(e) d'en finir avec la vie fasse l'objet d'une discussion détaillée qui prenne en compte tous les aspects, et intègre dans la mesure du possible l'environnement personnel du/ de la patient(e). L'opinion jusqu'à présent prévalente qu'il faut une maladie mortelle était et reste extrêmement arbitraire. D'une part, il existe actuellement des thérapies susceptibles de soigner des maladies jusqu'à

Il faut que le souhait d'un(e) patient(e) d'en finir avec la vie fasse l'objet d'une discussion détaillée qui prenne en compte tous les aspects.

présent mortelles, et d'autre part, il n'est défini nulle part à quelle distance de la mort naturelle prévue un suicide assisté est autorisé. La formulation choisie désormais est donc plus appropriée et plus concrète: les souffrances du/de la patient(e) sont déterminantes, et non l'estimation du médecin. L'«autorisation» paternaliste est remplacée par un «soutien» partenarial. L'objection selon laquelle cette directive donne trop d'importance à l'autonomie du/de la patient(e) d'un point de vue hédoniste n'est définitivement pas valable. Quiconque a connu les souffrances d'un(e) patient(e) gravement atteint(e) et sans espoir de guérison se représentera facilement que même dans une telle situation, la décision de mettre un terme à sa vie reste particulièrement dure. L'une des missions les plus importantes du médecin est de soutenir son/sa patient(e) dans les situations les plus difficiles. La direc- 
tive de l'ASSM permet à ce médecin qui accepte librement et conformément à ses convictions personnelles (et uniquement à lui), d'assumer la démarche d'accompagner le/la patient(e) sur cette voie difficile.

\section{La véhémence avec laquelle la FMH s'élève contre cette disposition est étonnante.}

La véhémence avec laquelle la FMH s'élève contre cette disposition est étonnante. D'une part, la fédération est représentée au Comité de direction de l'ASSM, et d'autre part, elle a participé à la consultation, c'est-àdire qu'elle a en permanence été impliquée dans l'ensemble du processus. Si la plupart des organisations sont en faveur de cette directive, il est alors conforme aux us et coutumes en Suisse qu'elle soit mise en œuvre ainsi. Exiger que tous reprennent l'opinion de la FMH est pour le moins surprenant. Et remettre ainsi en question toute la directive est juste irresponsable.

Exiger une nouvelle base légale là où elle n'est pas nécessaire soulève la question de savoir quel devrait être le but de cette intervention. D’autant plus que les responsables politiques à tous les niveaux ont clairement indiqué qu'ils ne légiféreront pas et ne modifieront aucune loi sur le sujet. Le refus d'accepter la directive mènera seulement à sa non-inscription dans le Code de déontologie. Elle sera tout de même valable, au plus tard lors du premier jugement par un tribunal.

L'ASSM est un partenaire essentiel du corps médical. Les réflexions éthiques indépendantes de la politique (professionnelle) et des assurances sont indispensables à la qualité et à la dignité de notre système de santé. Vouloir décider des thèmes et des positions de l'ASSM créerait un système dont nous ne voulons pas.

mfe Médecins de famille et de l'enfance Suisse soutient la directive sur l'attitude face à la fin de vie et à la mort dans sa globalité.

mfe Médecins de famille et de l'enfance Suisse soutient la directive sur l'attitude face à la fin de vie et à la mort dans sa globalité, consciente que ces problèmes sont difficiles et exigeants pour toutes les personnes concernées.

\section{Sujet d'actualité en ligne} www.bullmed.ch/fr/tour-dhorizon

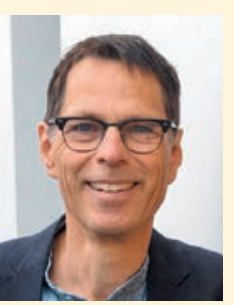

Interview vidéo d'Adrian Schmid, responsable du secrétariat d'eHealth Suisse

\section{Test d'endurance du dossier électronique du patient (DEP)}

Pendant une semaine à Berne, divers informaticiens ont collaboré pour tester à fond le DEP dans des conditions pratiques.

Marcel Marti, lic. phil. hist., Responsable politique et communication / Directeur adjoint de l'ASMAC

\section{«Plus de médecine et moins de bureaucratie!»- constructif et concret}

Moins de bureaucratie pour plus de temps au chevet du malade. 